ORIENTAÇÕES POLITICAS E PRÁTICA PROFISSIONAL EM INSTITUIÇÕES DE LONGA PERMANENCIA PARA IDOSOS

Joyce Alonso Canto da Silva ${ }^{1}$

Maria Helena Morgani de Almeida²

resumo

No Brasil, verifica-se aumento das doenças crônicas não transmissíveis que podem comprometer funcionalidade, autonomia e independência do idoso e resultar em institucionalização de permanência. Políticas públicas para idosos têm buscado garantir atenção integral e humanizada a essa população. A diversificação e capacitação de recursos humanos são importantes indicadores de implementação dessas políticas e, fundamentais para a prestação do cuidado integral e humanizado aos idosos. Supõe-se, entretanto, que recursos humanos orientados exclusivamente por políticas específicas possam não responder a complexidade das demandas assistenciais e institucionais, especialmente em Instituições de Longa Permanência para Idosos (ILPIs). O estudo investiga possí-

\footnotetext{
1 Terapeuta Ocupacional graduada pela Faculdade de Medicina da Universidade de São Paulo (FMUSP). Aprimoranda no Programa de Atendimento Interdisciplinar em Gerontologia e Geriatria do Instituto de Assistência Médica ao Servidor Público Estadual (IAMSPE). E-mail: joyce.canto@usp.br 2 Terapeuta Ocupacional graduada pela Universidade Federal de São Carlos (UFSCar). Profa. Dra. do Curso de Terapia Ocupacional do Departamento de Fisioterapia, Fonoaudiologia e Terapia Ocupacional da Faculdade de Medicina da Universidade de São Paulo (FMUSP). E-mail: hmorgani@usp.br
} 
veis semelhanças e diferenças entre práticas profissionais em ILPIs e orientações políticas presentes em documentos oficiais. Realizouse pesquisa documental entre 1989 e 2009, referente à publicação dos principais documentos oficiais e também pesquisa bibliográfica relativa às práticas assistenciais em ILPIs. Foram localizadas publicações de 1985 a 2010. Entre os 12 trabalhos sobre práticas assistenciais selecionados, 6 utilizam como referência ao menos 1 dos 9 documentos oficiais analisados. Cinco desses trabalhos dialogam com tais documentos através de discussões e 1 os utiliza somente para justificar sua prática. Ainda que de forma não sistemática, foi observada influência dos documentos oficiais sobre parte dos trabaIhos realizados. Nesses casos, os profissionais demonstraram utilizar dispositivos legais como instrumentos factíveis para o desenvolvimento de ações em saúde. Reforça-se a importância da ampliação do diálogo entre políticas e práticas profissionais, voltada à criação de políticas de amparo aos idosos residentes nas ILPIs e ao aprimoramento dos profissionais que atuam nessas instituições.

palavras - chave

Instituição de Longa Permanência para Idosos. Políticas Públicas.

Prática Profissional.

\section{Envelhecimento populacional e políticas públicas}

O envelhecimento populacional é um fenômeno mundial. As projeções mais conservadoras indicam que em 2020 haverá um contingente superior a 30 milhões de pessoas idosas em nosso país. Esse fenômeno acarreta crescimento das doenças crônicas não transmissíveis (RAMOS, 2005). Essas doenças podem ocasionar comprometimento da capacidade funcional com perda de autonomia e independência dos idosos (LEMOS; MEDEIROS, 2002) e resultar em hospitalização e institucionalização de permanência.

O envelhecimento populacional nos orienta em direção a um novo paradigma de saúde, para o qual o conceito de capacidade funcional é central (RAMOS, 2005). A partir desse conceito, um sujeito pode ser caracterizado como saudável, ainda que apresente uma ou mais doenças, desde que as mesmas não repercutam sobre sua independência e qualidade de vida.

Nessa perspectiva, o objetivo principal do sistema de cuidados deve ser a preservação da capacidade funcional do idoso, mantendo-o independente e 
na comunidade pelo maior tempo possível (RAMOS, 2005). Políticas públicas voltadas aos idosos, em nosso país, têm se voltado prioritariamente a esse mesmo propósito.

A Constituição brasileira de 1988 foi a primeira a tratar o idoso e a velhice como um problema social, avançando para além da assistência previdenciária e assegurando a proteção na forma de assistência social (MARTINS et al., 2007). Em 1994, no Brasil, a Lei no 8.842 estabeleceu a Política Nacional do Idoso (PNI) tendo como finalidades assegurar promoção da autonomia, integração e efetiva participação do idoso na sociedade. Para colocar em prática as ações preconizadas pela PNI, criou-se em 1997, o Plano de Ação Conjunta Governamental (RODRIGUES, 2007).

Já em 1998, a Organização Pan-Americana de Saúde (OPAS) realizou a XXV Conferência Sanitária, na qual se estabeleceram recomendações para o desenvolvimento de políticas, planos e programas de atenção aos idosos (SAYEG et al., 2002). Em 1999, estabeleceu-se a Política Nacional de Saúde do Idoso (PNSI), em conformidade com as diretrizes de promoção do envelhecimento saudável, manutenção da capacidade funcional, capacitação de recursos humanos especializados, apoio ao desenvolvimento de cuidados informais e apoio a estudos e pesquisas (SAYEG et al., 2002).

Com a Portaria no 73 do Ministério da Previdência e Assistência Social, em 2001, foram estabelecidas as Normas de Funcionamento de Serviços de Atenção ao Idoso no Brasil. A portaria propõe mudanças nas várias modalidades dos serviços, definindo parcerias, integração intersetorial e co-responsabilidade do governo, da sociedade e da família (SAYEG et al., 2002).

A seguir, elaborou-se o Plano Internacional de Ação sobre o Envelhecimento (PIAE), resultado da segunda assembleia mundial sobre envelhecimento (ONU, 2002). Esse se fundamenta em três princípios: Participação ativa dos idosos na sociedade, no desenvolvimento, na força de trabalho e na erradicação da pobreza; Promoção de saúde e bem-estar na velhice; e Criação de um ambiente propício e favorável ao envelhecimento (RODRIGUES et al., 2007). Em 2003, através da Lei no 1.074 , sancionou-se no Brasil o Estatuto do Idoso que, como instrumento legal, culminou com ações concretas contra a violência e os maus-tratos à pessoa idosa (SAYEG et al., 2002).

Em 2005, a Organização Mundial da Saúde (OMS) apresentou um documento que versou sobre um novo paradigma de entendimento do envelhecimento, intitulado "Envelhecimento Ativo: Um Marco para elaboração de Políticas". Esse texto apresenta os principais desafios a serem enfrentados no mundo, relacionados ao envelhecimento da população, e destaca o fato de que a saúde só pode ser criada e mantida com a participação de vários 
setores. O documento é o marco teórico da abordagem multidimensional focada na capacidade funcional, como novo paradigma (SAYEG et al., 2002).

No Brasil, em 2005, o Ministério da Saúde lançou sua Agenda de Compromisso pela Saúde e, nesse ano, o Pacto em Defesa da Vida apresentou como uma de suas prioridades a Atenção à Saúde do Idoso (RODRIGUES, 2007). Nesse mesmo ano, o Ministério da Saúde e a Agencia Nacional de Vigilância Sanitária (ANVISA), por intermédio da Resolução de Diretoria Colegiada RDC n 283 , preencheram uma lacuna nas políticas de atenção à pessoa idosa ao estabelecer novas normas de funcionamento às Instituições de Longa Permanência para Idosos (ILPIs). Embora já em 1989 o Ministério da Saúde, através da Portaria no 810 , tivesse apresentado Normas para o funcionamento de casas de repouso, clínicas geriátricas e outras instituições destinadas ao atendimento de idosos, entende-se que os idosos residentes nestas compõem uma população fragilizada e que certamente trata-se do segmento mais excluído da população idosa, necessitando da ação permanente do Estado para a proteção de seus direitos humanos e promoção de sua cidadania (SAYEG et al., 2002).

Por fim, em 2006, no âmbito da saúde, a política nacional foi atualizada e denominada Política Nacional de Saúde da Pessoa Idosa (PNSPI). Essa considera o Pacto pela Saúde e suas diretrizes operacionais para consolidação do SUS, reafirmando a necessidade de enfrentamento dos desafios impostos pelo processo de envelhecimento (MARTINS et al., 2007). Apresenta-se, a seguir, através de uma linha do tempo, síntese dos principais marcos políticos. 


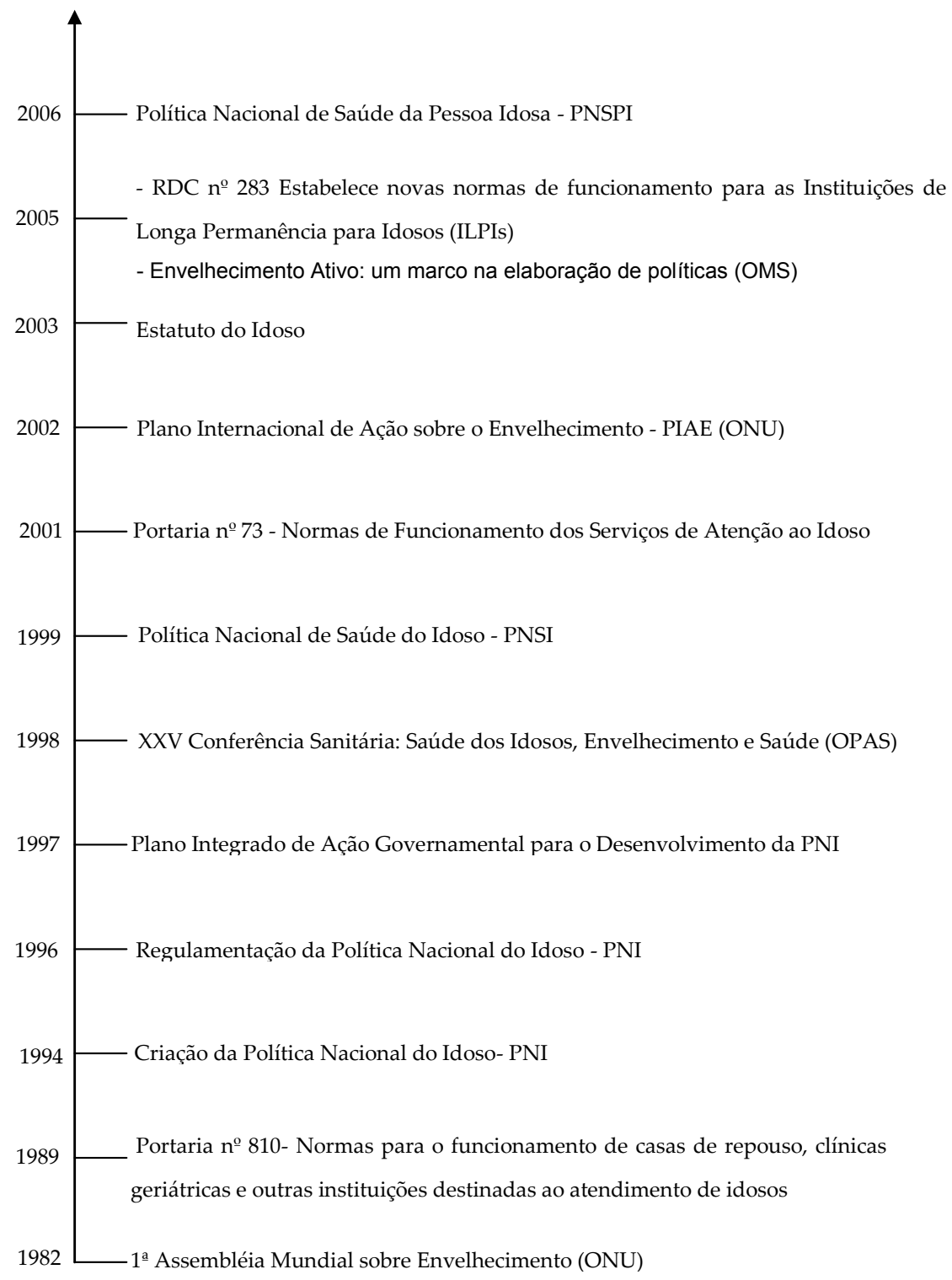


Embora documentos anteriores afirmem que as instituições geriátricas possam ter denominações diversas, tais como abrigo, asilo, lar, casa de repouso e clínica geriátrica, em 2005, a Resolução da Diretoria Colegiada, RDC nº 283, passa a vigorar, estabelecendo novas normas de funcionamento para esta modalidade assistencial e adotando um termo único para sua designação: Instituição de Longa Permanência para Idosos (ILPIs). Nesse documento, ILPIs são instituições governamentais ou não governamentais, de caráter residencial, destinadas ao domicílio coletivo de pessoas com idade igual ou superior a 60 anos, com ou sem suporte familiar, em condições de liberdade, dignidade e cidadania (POLLO; ASSIS, 2008).

A implementação dessa resolução torna-se um desafio para os órgãos fiscalizadores e as instituições, obrigados a se adequar à legislação e a superar o paradigma de atendimento enquanto caridade e assistencialismo, em direção ao atendimento como prestação de serviços, com qualidade e garantia dos direitos da pessoa idosa (POLLO; ASSIS, 2008).

A demanda pelo cuidado de idosos em instituições de longa permanência está aumentando no Brasil e no mundo, apesar das políticas públicas brasileiras afirmarem que o cuidado dos idosos é um dever da família em parceria com o Estado e que as ILPIs devam funcionar com base em critérios sociais. Segundo Camarano e Pasinato (2004), esse fato relaciona-se à procura pelas famílias por alternativas de atenção a seus familiares idosos com perdas de capacidade funcional resultantes de doenças crônicas, uma vez que a oferta de serviços adequados para pessoas nessa condição é ainda incipiente.

No contexto brasileiro, apontam-se os seguintes fatores de risco para internação de idosos: síndrome de imobilidade, múltiplos problemas clínicos, depressão, demência, alta hospitalar recente, incontinência, ser mulher, ter idade acima de 70 anos, ser solteiro, sem filhos, viúvo recente, morar sozinho, estar socialmente isolado e ser pobre (POLLO; ASSIS, 2007). Apesar desses fatores, os altos custos econômicos das instituições fechadas e, principalmente, os resultados, nem sempre positivos, propiciaram questionamentos a respeito dessa prática e a necessária atenção aos critérios precisos à sua indicação. Segundo Lemos e Medeiros (2002), as ILPIs são necessárias para atender o idoso pobre, sem família, sem casa para morar, ou mesmo que faz a opção de morar em asilo. 
Pollo e Assis (2008) afirmam que, para que as ILPIs ofereçam atendimento de qualidade, é fundamental assegurar aos profissionais que nela atuam espaços de supervisão, estudos, reuniões e qualificação continuada. Percebese, no entanto, que a capacitação dos profissionais da saúde para o cuidado geronto-geriátrico, incluindo aquele produzido nas ILPIs, é ainda incipiente para garantir a autonomia e independência dos idosos (MARTINS et al., 2007).

Na prática verifica-se a escassez de recursos humanos especializados para cumprir as diretrizes essenciais apresentadas pelo Estatuto do Idoso, tais como a promoção do envelhecimento saudável e a manutenção da capacidade funcional (MARTINS et al., 2007). Admite-se que, mesmo com a diversificação e capacitação de recursos humanos para atuarem de acordo com as políticas existentes, a prestação do cuidado integral e humanizado aos idosos em ILPIs não esteja assegurada. São reconhecidas a fragilidade e exclusão a que essa população está exposta, o que sugere a ampliação de referenciais e ações para responder aos desafios impostos pelas complexas demandas assistenciais e institucionais.

O presente estudo investiga possíveis semelhanças e diferenças entre práticas profissionais - produzidas em ILPIs e localizadas em bases de dados e sítios da internet - e orientações políticas presentes em documentos oficiais direcionados à população idosa.

\section{Método}

Optou-se por pesquisa descritiva de correlação, a qual segundo Gil (2002) consiste em estudar as influências de fenômenos entre si. Para realização da mesma, houve a aprovação do estudo pelo Comitê de Ética da Faculdade de Medicina da Universidade de São Paulo, sob o número 235/10 (aprovado em 15/09/10).

Foram definidas como variáveis de estudo: práticas profissionais nas ILPIs e orientações políticas voltadas a essas práticas. Para pesquisar essas variáveis, foi realizado levantamento bibliográfico preliminar com os termos: velhice, idosos, institucionalização de idosos, ILPIs, políticas para envelhecimento e normas de funcionamento, nas principais bases de dados na área da Saúde: Literatura Latino Americana e do Caribe em Ciências da Saúde LILACS, Scientific Eletronic Library on line - SCIELO, Biblioteca Virtual em Saúde - Bireme e Banco de Dados Bibliográficos da USP - Dedallus.

Devido ao reduzido número de trabalhos encontrados, ampliaram-se as fontes de referências. Para tanto, foi realizada uma pesquisa documental sobre 
políticas públicas e documentos oficiais dirigidos à população idosa e em seguida, pesquisa bibliográfica sobre práticas profissionais no cotidiano das ILPIs. A pesquisa documental englobou leis, decretos, portarias brasileiras, entre outros documentos da Organização Mundial da Saúde (OMS), Organização Panamericana da Saúde (OPAS) e Organização das Nações Unidas (ONU), dirigidos à população idosa. Nesses documentos, foram observadas diretrizes teóricas para ação profissional na área de saúde, incluindo aquelas praticadas no âmbito das ILPIs. Essa pesquisa se estendeu de 1989 - ano de publicação da Portaria no 810 que apresenta normas para o funcionamento de casas de repouso, clínicas geriátricas e outras instituições destinadas a idosos - até 2009, com a Tipificação Nacional de Serviços Socioassistenciais (BRASIL, 2009). Os documentos foram analisados em seu conteúdo por meio de análise temática.

A pesquisa bibliográfica sobre práticas profissionais em ILPIs compreendeu relatos de experiência, pesquisas teóricas, entre outras publicações incluídas no período de 1985 até 2010. Foram utilizadas como instrumentos de busca, as bases de dados on-line Sciello, Lilacs, Bireme além de outros sítios disponíveis na internet, tais como o Portal do Envelhecimento e o Portal da Sociedade Brasileira de Geriatria e Gerontologia (SBGG). Com base no DeCS (Descritores em Ciências de Saúde), o descritor utilizado foi ILPI, e como palavras-chave consideraram-se as denominações: casas de repouso, clínica geriá-trica, lar de idosos e asilo de idosos. Foram realizados cruzamentos desses termos com os seguintes: prática profissional, exercício profissional, ação profissional e intervenção. O resultado da busca forneceu inúmeros trabalhos que não incluíram relato ou reflexão sobre intervenções em ILPIs. Optou-se pela adoção de novos cruzamentos de termos, dessa vez envolvendo as áreas profissionais que podem atuar nas ILPIs: terapia ocupacional, fisioterapia, nutrição, assistência social, medicina, enfermagem, atividade física, musicoterapia, psicologia e fonoaudiologia. O resultado forneceu 38 trabalhos, e foram reduzidos para 12, após serem filtrados pelos seus resumos.

Esses trabalhos foram correlacionados e analisados com base nos documentos legais que antecedem a data de sua publicação. Realizou-se uma varredura eletrônica nos trabalhos e verificaram-se nesses, presença ou ausência das diretrizes teóricas presentes nos documentos oficiais, sem que fosse necessária a menção dos mesmos. Por fim, foi feita uma comparação entre as ações preconizadas nos documentos oficiais e aquelas mencionadas pelos trabalhos dos profissionais. 
Observou-se a escassez de documentos e políticas específicas direcionadas à população idosa residente nas ILPIs. Dessa forma, foram incluídos documentos que tratam dos direitos dos idosos em geral. Apenas 4 dos 9 documentos ocupam-se de questões referentes as ILPIs, sendo que desses, 3 regulamentam este tipo de serviço.

Destaca-se que as três principais políticas nacionais voltadas à população idosa: Política Nacional do Idoso (BRASIL, 1994), Política Nacional de Saúde do Idoso (BRASIL, 1999) e a Política Nacional de Saúde da Pessoa Idosa (BRASIL, 2006) voltam-se pouco para questões referentes à institucionalização dos idosos, sendo que a última não cita esta realidade.

Quatro documentos apresentam definições sobre o serviço de institucionalização dos idosos. A definição mais antiga, proveniente da Portaria 810 (BRASIL, 1989) se refere ao seu 'caráter de internato'. Assim também está posto na Portaria 73 (BRASIL, 2001). Porém, na definição apresentada pela RDC 283 (ANVISA, 2005) surgem os termos 'caráter residencial' e 'domicílio coletivo', complementados pela objetiva determinação das 'condições de liberdade, dignidade e cidadania'. Já a definição mais recente, oriunda da Tipificação Nacional dos Serviços Socioassistenciais (BRASIL, 2009), abstémse de caracterização dos aspectos citados, utilizando o termo 'acolhimento de idosos', embora também preconize o caráter residencial das ILPIs. Vê-se uma sensível e importante evolução do entendimento das ILPIs.

Interessante observar que, embora em 2005 o termo ILPI tenha sido proposto como único através da RDC 283 (ANVISA, 2005), nos documentos posteriores, outros termos continuam sendo empregados, como abrigo institucional e clínicas para idosos.

Percebe-se certa lacuna no que se refere ao público alvo do serviço. Sua indicação não é precisa. Embora os documentos apontem unanimemente que os idosos atendidos pelas ILPIs poderão ser dependentes ou independentes, a internação decorrente de vulnerabilidade social, ausência da família e mesmo como opção da família ou do próprio idoso não são discutidos.

Os objetivos do atendimento pelas ILPIs são apresentados de modo amplo e vago e incluem acolhimento, proteção, moradia e atenção.

Em relação ao funcionamento do serviço, observam-se apontamentos recorrentes referentes as ILPIs como locais de participação na comunidade 
em contraposição ao isolamento, conforme descritos no Estatuto do Idoso (2003), PIAE (ONU, 2002), na Portaria 810 (BRASIL, 1989), na RDC 283 (ANVISA, 2005) e na Portaria 73 (BRASIL, 2001).

Somente a Portaria 73 (BRASIL, 2001) apresenta uma classificação das ILPIs em três modalidades de acordo com o nível de dependêncialindependência para as atividades da Vida Diária.

Evidencia-se a falta de articulação e pouca consideração aos documentos já existentes na composição da legislação sobre o tema, com exceção da RDC 283 (ANVISA, 2005) que apresenta uma síntese das normas e diretrizes dos documentos precedentes.

Apenas o PIAE (ONU, 2002) e a Política Nacional do Idoso (BRASIL, 1994) citam a importância da formação profissional para o trabalho em ILPIs. A Política Nacional de Saúde da Pessoa Idosa (BRASIL, 2006) especifica a necessidade de formação na área da saúde da pessoa idosa mais amplamente.

Quanto à capacitação profissional, observam-se dois documentos que apresentam a Educação Permanente como instrumento de trabalho, sendo eles a Política Nacional de Saúde da Pessoa Idosa (BRASIL, 2006) e RDC n. 283 (ANVISA, 2005).

O trabalho em equipe é mencionado em quatro documentos, equipe interdisciplinar é preconizada pelo PIAE (ONU, 2002) e pela Política Nacional de Saúde da Pessoa Idosa (BRASIL, 2006); equipe multiprofissional é citada pela Portaria 810 (BRASIL, 1989); e equipe interprofissional, na Política Nacional do Idoso (BRASIL, 1994).

As diretrizes teóricas norteadoras das práticas assistenciais em ILPIs, em ordem decrescente de citação, foram: Atenção integral (Portaria 73, PNSI, Estatuto do idoso, PIAE, PNSPI, RDC 283, Tipificação Nacional dos Serviços Socioassistenciais), Manutenção e Promoção da capacidade funcional (PNSI, PNSPI). Humanização do cuidado (RDC 283, PNSPI); Promoção da qualidade de vida (Portaria 73 e PIAE); Promoção do envelhecimento saudável (PNSI); Promoção do envelhecimento ativo e saudável (PNSPI), Universalidade e equidade (PNSPI).

\subsection{Pesquisa bibliográfica}

A pesquisa capturou 12 trabalhos, a maior parte publicada nos últimos cinco anos, constituída, em sua maioria, por artigos publicados em revistas científicas, havendo alguns relatos de experiência provenientes de portais da internet e apenas uma dissertação. 
Dez trabalhos são provenientes de Instituições de Ensino Superior e somente 2 são de autoria de profissionais que têm suas atividades restritas à prática assistencial.

Metade dos trabalhos analisados cita políticas e documentos oficiais. Para amparar as discussões sobre a prática nas ILPIs, observa-se ainda a utilização de políticas e documentos não previstos pela pesquisa documental tai como: Regulamentação do Exercício Profissional da Enfermagem; Constituição 1988; Lei n. 8142, Participação da comunidade na gestão do Sistema Único de Saúde; Políticas de educação e desenvolvimento para o SUS, 2003; Política Nacional de Educação Permanente em Saúde, 2004 e Manual de funcionamento para instituição de longa permanência para idosos, SBGG 2003.

Em geral, os trabalhos se amparam em referências do campo de conhecimento do autor. Observa-se a recorrência de referências na área de Gerontologia e Enfermagem.

Dos 6 trabalhos que adotam políticas e documentos oficiais, 5 as utilizam não somente para justificar suas propostas, mas vão além e discutem suas práticas com base nesses referenciais. Todos são da área de enfermagem, tratando-se de quatro artigos e uma dissertação.

Os dois trabalhos provenientes de profissionais fora do circuito das Instituições de Ensino Superior, provêm das áreas de Medicina da Reabilitação, Serviço Social e Terapia Ocupacional e não utilizam as políticas e os documentos oficiais para embasarem ou refletirem sobre suas práticas.

\subsection{Análise comparativa}

Buscou-se verificar se os 6 trabalhos que se amparam em políticas e documentos oficiais adotam aqueles mais próximos cronologicamente às suas publicações. Mello e Padilha (2000) amparam-se na legislação brasileira com o intuito de justificar a importância do tema "maus tratos no cuidado à população idosa". Os autores publicam no ano de 2000 e adotam legislação atual já que o conjunto de documentos utilizados data de 1989 até 1999 . O trabalho de Moraes e Duarte data de 2002, nele os autores mencionam documentos de 1994 a 1999, mas não a Portaria n. 73 publicada em 2001. Learndt et al. publicam em 2006 e mencionam políticas situadas entre 1986 e 2003, não citando a RDC n. 283. Silva et al. têm trabalho datado de 2008 e utilizam documentos oficiais que datam desde 1986 até 2006, o que demonstra atualização de sua publicação. Outro trabalho da mesma autora com novos colaboradores, publicado em 2009 utiliza como referências, documentos oficiais de 
2005 a 2006, mantendo-se, portanto, devidamente atualizados. Da mesma forma, Passador publica seu trabalho em 2008 utilizando, para tanto, documentos oficiais bastante atuais, datados entre 1986 e 2006. Pode-se afirmar, assim, que os trabalhos mantiveram-se sincronizados com documentos referentes às suas datas de publicação. Nota-se, ainda, que alguns trabalhos utilizam um rol diversificado e amplo de documentos, reforçando seus argumentos e enriquecendo suas discussões.

As discussões travadas pelos autores com as referências documentais concentram-se na ação dos profissionais da enfermagem junto à população idosa residente nas ILPIs. Considera-se, contudo, que se tratam de discussões aplicáveis ao cuidado gerontológico exercido pelas variadas profissões da saúde, uma vez que englobam temas como educação permanente, cuidado não homogenizador e formação profissional.

Quanto às diretrizes teóricas para as práticas, destacam-se qualidade de vida, sendo citada por quase todos os trabalhos; atenção integral e capacidade funcional, citada por metade deles e humanização mencionada somente em 2 publicações.

As ações preconizadas pelas políticas e documentos oficiais são variadas e incluem construção de projetos de vida, participação em grupos de convivência, informação para prática de sexo seguro e prevenção de isolamento social. Muitas dessas ações estão presentes nas publicações dos profissionais das ILPIs, destacando-se ações voltadas para reabilitação, física e cognitiva, e convivência entre os residentes, enquanto nos documentos, as atividades centradas na integração do idoso com a comunidade têm maior destaque.

Observam-se ações que estão preconizadas nas políticas e não aparecem na prática, como as ações de integração com a comunidade e de acesso aos direitos. Por sua vez, há ações que se desenvolvem nas práticas, mas não foram previstas pelas políticas, tais como ações desenvolvidas a partir da escuta dos trabalhadores e intervenções voltadas aos cuidadores e familiares.

\section{Discussão}

Muitos documentos estabelecem que o cuidado dos idosos dependentes deva ser responsabilidade das famílias. Porém, fatores como a redução da fecundidade, crescente participação da mulher no mercado de trabalho, aumento da sobrevivência de pessoas com redução da capacidade física, cognitiva e mental aumentam a necessidade de compartilhar o cuidado ao idoso (CAMARANO; KANSO, 2010). 
Para Vieira (2003), a falta de apoio de uma rede de serviços geriátricos de suporte, em especial às famílias sem recursos socioeconômicos, imporá a institucionalização como alternativa única para atender às demandas de seus idosos. A despeito dessas evidências, as três mais importantes políticas públicas nacionais dirigidas às pessoas idosas não fornecem orientações direcionadas aos cuidados de idosos em ILPIs.

A pesquisa documental, analisada cronologicamente, parece demonstrar uma importante mudança na compreensão das ILPIs. Surge paulatinamente a ideia de que a instituição não pode ser um internato, mas sim uma residência, um domicílio coletivo. Noções como a de privacidade, liberdade e cidadania também surgem nos documentos mais recentes, o que evidencia mais uma vez a sensível evolução.

Observa-se que normatizações se concentram em aspectos físicos e legais, sendo que os objetivos, o público alvo e o funcionamento das ILPIs aparecem como menos importantes. Talvez, ainda estejamos num momento em que o essencial seja garantir padrões mínimos de funcionamento dessas instituições.

Considerando-se os profissionais que trabalham nas ILPIs co-responsáveis pela implantação e implementação de políticas públicas para o setor, aponta-se a necessidade de sua capacitação continuada. De acordo com Rodrigues e Rauth (2002), enquanto não houver recursos humanos treinados especialmente para atender a população idosa, não é possível haver atenção integral, digna e eficaz. São necessários profissionais conhecedores da realidade social e de saúde dessa população, das tecnologias existentes, dos recursos disponíveis e dos dispositivos legais como instrumentos para o desenvolvimento de ações críticas e construtivas (MARTINS et al., 2007).

Dentre a totalidade de trabalhos captados, a metade cita políticas e documentos oficiais e promove discussões sobre as práticas assistenciais a partir deles, o que pode sugerir que esses documentos estimulam boa parte dos profissionais a refletirem sobre suas ações. Há, porém, profissionais no presente estudo que não se mostraram sintonizados com tal importância, descrevendo intervenções pertinentes e interessantes sem, no entanto, travar discussões críticas sobre as mesmas.

As ações identificadas, a partir das revisões, são variadas e observa-se tendência à concretização da integralidade na atenção ao idoso em todas as dimensões da vida. Destaca-se, entretanto, a ausência de recomendações para o cuidado no processo da morte digna, com os direitos preservados.

Quanto aos serviços mais frequentes nas instituições brasileiras, Camarano e Kanso (2010) constatam que os serviços médicos e de fisioterapia são encontrados em $66,1 \%$ e $56,0 \%$ delas, respectivamente. Outras atividades 
com a função de promover integração entre os residentes e facilitar o exercício de papéis sociais, como lazer e cursos diversos são menos frequentes, declarados por menos de $50 \%$ das instituições pesquisadas.

Os resultados da presente pesquisa bibliográfica se aproximam muito dessas proporções, o que nos faz pensar que embora o reduzido número de trabalhos encontrados tenha sido um fator limitante, aproximações com dados da realidade cotidiana foram possíveis.

\section{Conclusão}

Este estudo explorou as formas de diálogo que têm sido estabelecidas entre documentos oficiais e práticas profissionais em ILPIs. Por meio de comparações entre tais publicações, foram observadas semelhanças e diferenças. Notou-se que alguns profissionais demonstraram utilizar os dispositivos legais como instrumentos factíveis para o desenvolvimento de ações em saúde, sendo que as ações por eles desenvolvidas guardam sensível relação com as aquelas preconizadas pelos documentos oficiais. Em contrapartida, observaram-se ações que estão preconizadas nas políticas e não se desenvolveram na prática e, ações praticadas que não foram previstas pelas políticas.

Admitem-se limitações do estudo, uma vez que práticas assistenciais não foram identificadas na amplitude que se pretendia. Sugerem-se estudos empíricos que possam ampliar o conhecimento das ações desenvolvidas por profissionais em ILPIs. Apesar das limitações apontadas, exploraram-se questões cruciais relativas ao diálogo estabelecido entre orientações políticas e práticas profissionais nas ILPIs.

Com base nas semelhanças e diferenças entre o preconizado e o realizado, sugere-se ampliação do diálogo entre usuários, profissionais, gestores de serviços e formuladores de políticas. Considera-se que esse diálogo deva encaminhar-se a criação de políticas de amparo aos idosos residentes nas ILPIs, voltadas à prevenção e reversão de fragilidade, melhora da capacidade funcional, promoção da inclusão social, atenção integral e humanizada aos idosos em ILPIs, bem como ao aprimoramento e qualificação dos seus profissionais. 
abstract

In Brazil there is an increase in chronic diseases that can compromise the functionality, autonomy and independence of the elderly and result in his permanent institutionalization. Public policies for the elderly have sought to guarantee comprehensive and humanized care of the elderly population. Diversification and training human resources are important indicators of implementation of these policies and fundamental to the provision of comprehensive and humanized care for the elderly. It is assumed, however, that human resources geared exclusively by specific policies may not respond to the complexity of care and institutional demands, especially in Homes for the Aged. This study investigates possible similarities and differences between professional practices conducted in Homes for the Aged and policy guidelines of official documents. A documentary research was carried out between 1989 and 2009 on the main publication of official documents and also literature research was carried on care practices in Homes for the Aged. Publications were found dating from 1985 to 2010. Among the 12 selected papers on care practices, 6 use as a reference, at least one of the nine official documents analyzed. Five of these works relate to such documents through discussions and one uses them only to justify their practice. Albeit not systematically, the influence of official documents on the part of the work was observed. In such cases, practitioners demonstrated using such legal devices as feasible instruments for the development of actions in health. It reinforces the importance of expanding the dialogue between policy and practice professionals, focused on the creation of policies to support elderly residents in Homes for the Aged and improvement of professionals working in these institutions.

keywords

Homes for the Aged. Public Policies. Professional Practice. 
AGENCIA NACIONAL DE VIGILÂNCIA SANITÁRIA (ANVISA). Resolução da Diretoria Colegiada n²83, de 26 de setembro de 2005. Aprova o regulamento técnico que define normas de funcionamento para as instituições de longa permanência para idosos. Diário Oficial da União, Brasília, DF, 2005.

BRASIL. Ministério da previdência e assistência social. Portaria n 73 , de 10 de maio de 2001. Aprova as Normas de funcionamento de serviços de atenção ao idoso no Brasil. Diário Oficial da União, Brasília, DF, 2001.

BRASIL. Ministério da Saúde. Portaria n 395, de 10 de dezembro de 1999. Aprova a Política de Saúde do idoso. Diário Oficial da União, Brasília, DF, 1999.

BRASIL. Ministério da Saúde. Portaria no 2528, de 19 de outubro de 2006. Aprova a Politica Nacional de Saúde da Pessoa Idosa. Diário Oficial da União, Brasilia, DF, 2006.

BRASIL. Ministério da Saúde. Portaria n 810, de 22 de setembro de 1989. Aprova as normas e padrões para o funcionamento de casas de repouso, clínicas geriátricas e outras instituições destinadas ao atendimento de idosos: Diário Oficial da União, Brasilia, DF, 1989.

BRASIL. Ministério Do Bem-Estar Social. Lei n8842, de 04 de janeiro de 1994. Dispõe sobre a política nacional do idoso, cria o Conselho Nacional do Idoso e dá outras providências. Diário Oficial da União, Brasília, DF, 1994.

BRASIL. Ministério do Desenvolvimento Social e Combate à fome. Conselho nacional de Assistência Social. Resolução no 109, de 11 de novembro de 2009. Aprova a Tipificação Nacional de Serviços Socioassistenciais. Diário Oficial da União, Brasilia, DF, 2009.

CAMARANO, Ana Amélia; KANSO, Solange. As instituições de longa permanência para idosos no Brasil. Revista Brasileira de Estudo de População, Rio de Janeiro, v. 27, n. 1 , p. 233-235 jan./jun. 2010.

CAMARANO, Ana Amélia; PASINATO, Maria Tereza. O envelhecimento da populacional na agenda das políticas públicas. In: CAMARANO, Ana Amélia (Org). Os novos idosos brasileiros muito além dos 60? Rio de Janeiro: IPEA - Instituto de Pesquisa Econômica Aplicada, 2004, p. 253-292.

GIL, Antonio Carlos. Como Elaborar Projetos de Pesquisa. 4. ed. São Paulo: Atlas, 2002.

LEARNDT, Maria Helena; WILLIG, Mariluci Hautsch; SILVA, Sheilla Cristina da; SHIMBO, Adriano Yoshio; TALLMANN, Ana Elisa Casara; MARUO, Gláucia Harume. O Idoso institucionalizado e a cultura de cuidados profissionais. Cogitare Enfermagem, Curitiba, v. 11, n. 2, p. 117-123, mai./ago. 2006.

LEMOS, Naira Dutra; MEDEIROS, Sonia Lima. Suporte Social ao Idoso Dependente. In: FREITAS, Elizabete Viana de et al. (Orgs) Tratado de Geriatria e Gerontologia. 2. ed. São Paulo: Guanabara Koogan, 2002, p. 893-899.

MARTINS, Josiane De Jesus; SCHIER, Jordelina; ERDMANN, Alacoque Lorenzini; ALBUQUERQUE, Gelson Luiz de. Políticas públicas de atenção à saúde do idoso: reflexão acerca da capacitação dos profissionais da saúde para o cuidado com o idoso. Revista Brasileira de Geriatria Gerontologia. Rio de Janeiro v.10, n. 3, p. 371-382. ago./set., 2007.

MELLO, Ana Lúcia Schaefer Ferreira de; PADILHA, Dalva Maria Pereira. Instituições Geriátricas e Negligência Odontológica. Revista da Faculdade de Odontologia de Porto Alegre, Porto Alegre, v. 41, n 1, p. 44-48, jul. 2000.

MORAES, Izimar De Amorim Louzada; DUARTE, Maria Jalma Rodrigues Santana. Desinstitucionalizando o cuidar: uma reflexão voltada para as necessidades do idoso asilado. Revista de enfermagem. UERJ, Rio de Janeiro, v. 10, n. 3, p 237-242, set/ dez. 2002. 
ORGANIZAÇÃO DAS NAÇÕES UNIDAS. Plano de Ação Internacional Sobre o Envelhecimento. Madri, 2002.

PASSADOR, Mirela Berloti. Identificação dos cuidados à saúde prestados, pela equipe de enfermagem, aos idosos residentes em uma instituição de longa permanência filantrópica do município de São Paulo. Faculdade de Saúde Pública da Universidade de São Paulo, 2008. 98f. Dissertação (Mestrado em Serviços de Saúde Pública) São Paulo, 2008.

POLLO, Sandra Helena Lima; ASSIS, Mônica de. Instituições de Longa Permanência para Idosos - ILPIS: Desafios e alternativas no município do Rio de Janeiro. Revista Brasileira de Geriatria e Gerontologia, Rio de Janeiro, v. 11, n.1, p. 29-44, nov./dez., 2008.

RAMOS, Luiz Roberto. A Mudança de Paradigma na Saúde e o Conceito de Capacidade Funcional. In: SUMSION, Thelma. Guia de Medicina Ambulatorial e Hospitalar. São Paulo: Manole, 2005, p. 1-7.

RODRIGUES, Nara Costa; RAUTH, Jussara. Os Desafios do Envelhecimento no Brasil. In: FREITAS, Elizabete Viana de et al. (Orgs.). Tratado de Geriatria e Gerontologia. 2. ed. São Paulo: Guanabara Koogan, 2002, p. 189-192

RODRIGUES, Rosalina Aparecida Partezani; KUSUMOTA, Luciana; MARQUES, Sueli; FABRÍCIO, Suzele Cristina Coelho; RASSET-CRUZ, Idiane; LANGE, Celmira. Política Nacional de Atenção ao Idoso e a Contribuição da Enfermagem. Texto Contexto de Enfermagem. Florianópolis, v. 16, n. 3, p. 536-545, jul./set. 2007.

SAYEG, Mário Antônio; MESQUITA, Regina Ângela Viana; COSTA, Neidil Espínola. Políticas Públicas de Saúde para o Envelhecimento. In: FREITAS, Elizabete Viana de et al. (Orgs). Tratado de Geriatria e Gerontologia. 2. ed. São Paulo: Guanabara Koogan, 2002, p. 1394-1401.

SILVA, Bárbara Tarouco da; BARLEM, Edison Luiz Devos; LUNARDI, Valeria Lerch; SANTOS, Silvana Sidney Costa. Educação Permanente: Instrumento de Trabalho do Enfermeiro na Instituição de Longa Permanência. Ciência, Cuidado e Saúde, Maringá, v. 7, n. 2, p 256-261, abr./jun. 2008

SILVA, Barbara Tarouco; SANTOS, Silvana Sidney Costa: SILVA Mara Regina Santos da: SOUSA, Lenice Dutra de. Percepção das pessoas idosas sobre a institucionalização: reflexão acerca do cuidado de enfermagem. Revista de Enfermagem do Nordeste, Fortaleza, v. 10, n. 4, p. 118-25, out/dez. 2009.

VIEIRA, Eliane Brandão. Instítuições Geriátricas: Avanço ou Retrocesso? Rio de Janeiro: Revinter, 2003.

Recebido em: 24/01/2012

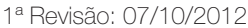

Aceite Final: 26/10/2012 
